\title{
RELATIONSHIP BETWEEN OXIDANT AND ANTIOXIDANT ENZYMES STATUS IN TYPE 2 DIABETIC PATIENTS WITH NEPHROPATHY IN SAUDI POPULATION
}

\author{
RAMPRASAD NAGARAJRA0*, SAMIR ABDULKARIM ALHARBI \\ Department of Medical Laboratory Science, College of Applied Medical Sciences, Shaqra University, Al-Quwayiyah, Kingdom of Saudi \\ Arabia. Email: ramprasad7u@gmail.com
}

Received: 06 October 2017, Revised and Accepted: 06 November 2017

\begin{abstract}
Objective: Oxidative stress has crucial role in pathogenesis of diabetic complications. Diabetic nephropathy (DN) is an important microvascular complication of diabetes and is widely recognized as the most common cause of the end-stage renal disease (ESRD) seen in clinical practice. Chemically, oxidative stress occurs as a result of increased levels of lipid peroxides and free radical intermediates, as well as a decrease in the total antioxidant capacity. This study analyzed the relationships between oxidant and antioxidant markers of DN in patients with type 2 diabetes mellitus (T2DM).
\end{abstract}

Methods: A descriptive study was taken during the period from November 2016 to August 2017. The present study included 53 patients suffering from T2DM without nephropathy and 51 patients T2DM with nephropathy along with 69 age- and sex-matched healthy controls. Various biochemical parameters, antioxidant enzymes, and malondialdehyde (MDA) levels were measured and compared.

Results: The glycosylated hemoglobin (HbA1c), urea, creatinine, microalbuminuria, and MDA levels were significantly increased (p<0.001), and antioxidant enzyme activities such as glutathione peroxidase (GPx), superoxide dismutase (SOD), and catalase (CTL) were significantly decreased $(\mathrm{p}<0.001)$ in T2DM with nephropathy patients followed by T2DM without nephropathy and control groups. There were a good negative correlations of HbA1c and GPx ( $\mathrm{r}=-0.79)$, SOD ( $\mathrm{r}=-0.61)$ and CTL $(\mathrm{r}=-0.74)(\mathrm{p} \leq 0.05)$, whereas there was an excellent positive correlation of MDA concentration $(\mathrm{r}=0.85, \mathrm{p} \leq 0.05)$ with HbA1c levels in diabetes with nephropathy.

Conclusion: The study illustrated that, in diabetic patients, there is an increased concentration of lipid peroxides which may contribute to decreased levels of cellular antioxidant enzymes, further leading to T2DM with nephropathy. Hence, monitoring of these antioxidant enzymes and microalbuminuria parameters in the early stage of diabetic patients could be vital importance in possible preventing further development of complications. We suggest potential and new multiproperty antioxidants therapy as one of the most important treatment strategies for diabetic patients without nephropathy for the prevention and slowing of diabetic with nephropathy before reaching to ESRD.

Keywords: Type 2 diabetes mellitus, Diabetic nephropathy, Oxidative stress, Antioxidant enzymes, Malondialdehyde, Glycosylated hemoglobin, microalbuminuria.

(C) 2018 The Authors. Published by Innovare Academic Sciences Pvt Ltd. This is an open access article under the CC BY license (http://creativecommons. org/licenses/by/4. 0/) DOI: http://dx.doi.org/10.22159/ajpcr.2018.v11i1.22940

\section{INTRODUCTION}

Diabetes mellitus (DM) is widely recognized as one of the leading causes of death and disability worldwide. The prevalence of DM will likely rise from $6 \%$ to over $10 \%$ in the next decade. In 2000 , the World Health Organization (WHO) recorded a total of 171 million people for all age groups worldwide ( $2.8 \%$ of the global population) who have DM, and the numbers are expected to rise to 366 million (4.4\% of the global population) by 2030 [1].

Oxidative stress is an imbalance between the systemic manifestation of reactive oxygen and nitrogen species (ROS) and the human biological system's ability to readily detoxify the reactive intermediates or to repair the resulting damage. Redox imbalance caused by increased ROS concentration and/or reduced antioxidant reserve leads to pathological consequences, including damage to proteins, lipids, and deoxyribonucleic acid (DNA). Thus, oxidative stress can cause disruptions in normal mechanisms of cellular signaling. Chemically, oxidative stress is associated with increased production of oxidizing species or a significant decrease in the effectiveness of antioxidants and antioxidant enzymes [2].

Hyperglycemia can increase oxidative stress through several pathways. The hypothesis is supported by evidence that many biochemical pathways strictly associated with hyperglycemia (glucoseautoxidation, polyol pathway, prostanoid synthesis, and protein glycation) can increase the production of free radicals. Furthermore, exposure of endothelial cells to high glucos leads to augmented production of superoxide anion $\left(\mathrm{O}_{2}^{-}\right)$and hydrogen peroxide $\left(\mathrm{H}_{2} \mathrm{O}_{2}\right)$, which may quench nitric oxide, a potent endothelium-derived vasodilator that participates in the general homeostasisof the vasculature. There are many evidence that oxidative stress plays a key role in the most pathogenic pathways of diabetic complications. Free radicals such as superoxide can induce cell and tissue injuries throughout lipid peroxidation (LPO) [3]

Diabetic nephropathy (DN) is an important microvascular complication of DM and is widely recognized as the most common cause of the end-stage renal disease (ESRD) seen in clinical practice. The kidneys have many tiny blood vessels that filter waste from the blood. High blood glucose and hypertension from DM can diminish the blood vessels, and afterward, kidney will not able to work and it completely stops functioning. The condition not only causes disability but also it is associated with a high mortality rate in diabetic patients as well [4]. Nephropathy complicates approximately $30 \%$ of type 2 diabetic patients. The development of vascular complications in DM correlates with the intensity of hyperglycemia. High intracellular glucose concentration has been suggested to be a prerequisite for the development of functional and structural changes in the kidney typical of DN [5]. 
Studies have shown that the extent of microalbuminuria affects longterm prognosis and the emergence of microalbuminuria not only indicates the early stage of DN but may also be the consequence of extensive damage to systemic vascular endothelial cells [6]. MDA is widely used as a marker of oxidative stress in many studies. MDA is a water-soluble low molecular weight and LPO product. Increased oxygenated compounds, particularly, aldehydes such as MDA are produced during the attack of free radicals on membranes, lipoprotein, and polyunsaturated fatty acids [7]. Assessment of LPO in the blood provides useful information for the prognosis of DN patients. There is no doubt that oxidative stress plays a pivotal or central role in body as well as the decrease in the protective total antioxidant capacity.

A good antioxidant status may be important for a healthy life of human beings. Low plasma levels of antioxidant as well as low intake of dietary antioxidants have been associated with an increased risk of so many chronic diseases [8]. Enzymatic glutathione peroxidase (GPx), superoxide dismutase (SOD), catalase (CTL), and chemical antioxidants play an important role in alleviating tissue damage due to the formation of free radicals and oxidants. Therefore, the aim of this study was to evaluate the status of oxidants and particularly key antioxidant enzymes in type 2 diabetes mellitus (T2DM) patients with and without nephropathy compared with normal healhy subjects.

\section{METHODS}

\section{Study area}

The descriptive study was taken from November 2016 to August 2017. The T2DM with and without nephropathy patients attending the Outpatient Department of Medicine and some patients were admitted to Medicine and Nephrology unit of the Al-Quwayiyah General Hospital, Kingdom of Saudi Arabia.

\section{Study subjects and groups}

Consecutive 104 patients with T2DM admitted to hospital were selected for the study, they were between 40 and 60 years old age, and they were further classified into 2 groups. Fifty-three patients had DM without nephropathy (group 2) of both the sexes (male 29 and 24 females), and fifity-one patients (male 27 and 24 females) had DM with nephropathy (group 3). The criteria for the diagnosis of T2DM was based on the 2006 WHO report [9], and the presence of early stage DN was based on the staging criteria proposed by Mogensen et al. [10]. Nephropathy is clinically defined by the presence of persistent proteinuria, microalbuminuria ( $>30 \mathrm{mg} /$ day) in DM patients in the absence of urinary tract disease, and on the basis of clinical history, physical examination, renal biopsy, and biochemical investigations. Smoking was defined as regular smoking of cigarettes/beedies (local type of tobacco). When those patients whose body mass index (BMI) was $>30 \mathrm{~kg} / \mathrm{m}^{2}$ were considered as obese. Sixty-nine healthy volunteers, both age- and gender-matched, who were non-diabetic subjects without nephropathy were considered as controls (group 1). Subjects suffering from hepatic disease, rheumatoid arthritis, autoimmune disease, strokes, cerebrovascular accidents, any chronic or acute inflammatory illness, pregnancy and lactating mothers, alcoholics, with juvenile T2DM and type $1 \mathrm{DM}$ and patients taking any kinds of multivitamin and lipid-lowering agents were excluded from the study.

\section{Methods of sample collection and biochemical assays}

Blood samples were obtained after an overnight fast. Five ml of plain blood and $3 \mathrm{ml}$ of EDTA blood were collected from each subject, and the serum was carefully separated and transferred to microtubes and stored at $+4^{\circ} \mathrm{C}$ before analysis. Anticoagulated blood samples were centrifuged at 4500 RPM for $15 \mathrm{~min}$. For collecting urine samples at $24 \mathrm{~h}$, wide-mouthed $3 \mathrm{~L}$ sterile containers were provided to the patients and control subjects, and instructions were given regarding urine collection. The urine volume was measured, and $10 \mathrm{ml}$ of sample was preserved for analysis. The fasting blood glucose levels were measured using fully automated biochemistry analyzers [11]. Glycemic control was assessed by measuring glycated hemoglobin using the resin-ion exchange method [12]. The serum urea and creatinine were measured by routine clinical chemical assays.

\section{Preparation of erythrocyte hemolysate}

After plasma separation, the buffy coat was discarded and packed red blood corpuscles (RBC) were washed 3-4 times with isotonic $0.9 \%$ saline solution $(\mathrm{pH}=7.4)$. The washed $\mathrm{RBCs}$ were hemolyzed by suspending in chilled distilled water, followed by centrifugation $(1400 \times g)$ at $4^{\circ} \mathrm{C}$. The supernatant was the hemolysate, which was directly used for estimation of GPx, SOD, and CTL activity [13]

\section{Estimation of GPx}

GPx activity catalyzes the oxidation of glutathione (GSH) by cumene hydroperoxide. In the presence of glutathione reductase (GR) and reduced nicotinamide adenine dinucleotide phosphate (NADPH), the oxidized glutathione is immediately converted to the reduced form with a concomitant oxidation of NAPH to NADP'. The decrease in absorbance at $340 \mathrm{~nm}$ is measured. GPx was measured by the method of Paglia and Valentine [14].

\section{Estimation of SOD}

The method employs xanthine and xanthine oxidase to generate superoxide radicals which react with 2-4-iodophenyl 3-4-nitrophenol5-phenyl tetrazolium chloride (INT) to form a red formazan dye. The SOD activity is then measured by the degree of inhibition of this reaction and measured by UV spectrophotometry, and absorbance was monitored at $505 \mathrm{~nm}$ for $3 \mathrm{~min}$. One unit of SOD is that which causes a $50 \%$ inhibition of the rate of reduction of INT under the condition of the assay [15].

\section{Estimation of CTL}

CTL was assayed by the method of Beers and Seizer [16]. The serum was incubated with $0.01 \mathrm{ml}$ of absolute ethanol at $4^{\circ} \mathrm{C}$ for $30 \mathrm{~min}$, after which $10 \%$ Triton X-100 was added so as to have a final concentration of $1 \%$. The sample, thus obtained, was used to determine CTL activity by measuring the breakdown of $\mathrm{H}_{2} \mathrm{O}_{2}$ spectrophotometrically at $240 \mathrm{~nm}$ and expressed as Bergmeyer units/g $\mathrm{Hb}$.

\section{Estimation of malondialdehyde (MDA)}

MDA was estimated by thiobarbituric acid (TBA) method. Autoxidation of unsaturated fatty acids involves in the formation of semi-stable peroxides which then undergo a series of reactions to form short chain aldehydes like MDA. One molecule of MDA reacts with two molecules of TBA with the elimination of water to yield pink crystalline pigment with a maximum absorption of $535 \mathrm{~nm}$ [17]

\section{Ethical approval}

The study was conducted with the approval of the Institutional Ethical and Human Research Committee. Written informed consent was also obtained from all the patients.

\section{Statistical evaluation}

The statistical analysis was undertaken using SPSS version 17.0 software. All values are expressed as mean \pm standard deviation. Differences between the means were calculated by analysis of variance test (multiple comparisons). Student's $t$-test was used to estimate the significant difference between the groups. Pearson's correlation analysis was used to test the correlation between various biochemical parameters. A p $<0.05$ was considered to statistically significant.

\section{RESULTS}

The clinical characteristics of two groups of patients and non-diabetic controls are presented in Table 1. In the present study, the numbers of patients with obesity and hypertensive was more on the T2DM with nephropathy group, compared to those with T2DM without nephropathy or controls, whereas smokers are similar in both groups.

Serum fasting blood glucose, glycosylated hemoglobin (HbA1c), urea, creatinine, and microalbuminuria were increased in T2DM 
without nephropathy (Table 2) and further significantly increased in T2DM with nephropathy patients compared to controls $(\mathrm{p}<0.001)$. Antioxidant enzyme activities such as GPx, SOD, and CTL were significantly decreased $(\mathrm{p}<0.001)$ in T2DM with nephropathy patients compared to T2DM patients without nephropathy and non diabetic controls. Significantly increased levels of MDA were observed in T2DM patients with nephropathy compared to normal subjects $(\mathrm{p}<0.001)$ as summarized in Table 3.

To assess the relationship between HbA1c levels with various antioxidant enzymes and oxidative stress factor, a linear correlation analysis was carried out, and Pearson's correlation coefficient was used. Analysis revealed that GPx, SOD, and CTL activities showed an inverse relationship with $\mathrm{HbA1c}$ level. MDA concentration was positively correlated with HbA1c levels in diabetic patients without nephropathy and those with diabetes and nephropathy as shown in Table 4

\section{DISCUSSION}

It has been suggested that free radicals are responsible for the development of DM macroangiopathy as well as microangiopathy. DN is one of the most important microvascular complications of DM and a major cause of ESRD. Several mechanisms are thought to be involved in the pathogenesis of DN and its complications, and some of these pathways are: Formation of advanced glycation end products, polyol pathway activation, hexosamine biosynthetic pathway, activation of protein kinase $\mathrm{C}$, and then, stimulating NADPH oxidase to induce ROS generation $[18,19]$. ROS activates signal transduction cascades and transcription factors leading to transcriptional activation of profibrotic genes. Further, PKC, transforming growth factor- $\beta$ (TGF- $\beta$ ), and intrarenal rennin angiotensin aldosterone system stimulated by hyperglycemia-induced ROS, in turn, these ROS are activated and involve in glomerular mesangial expansion and tubulointerstitial fibrosis. It is now clear that overproduction of ROS in T2DM is a direct consequence of hyperglycemia, and various types of cells including endothelial, vascular smooth muscle, mesangial and tubular epithelial cells are capable of producing ROS under glycemic condition [20]. TGF- $\beta$ plays an important role in the development of renal hypertrophy and accumulation of extracellular matrix components in DM. The expression of TGF- $\beta$ was found to increase in DN of experimental animal and in human studies [21]. Interestingly, oxidative stress has been suggested as a common product at the level of LPO, protein oxidation, and DNA damage is involved in the pathogenesis of DN in association with T2DM.

DN is one of the common complications of T2DM and has a leading cause of ESRD in many countries. It is believed that the pathophysiological mechanisms of renal disorder are similar in both types of diabetes. The pathogenesis of clinical course of DN can be monitored by structural and hemodynamic changes. The earliest changes are an increase in glomerular filtration rate, also call "hyperfiltration" stage, which is followed by detectable glomerular lesions with normal albumin excretion rate. The next change is the development of microalbuminuria.

Table 1: Clinical details of the study subjects

\begin{tabular}{|c|c|c|c|}
\hline \multirow[t]{2}{*}{ Particulars } & \multicolumn{3}{|l|}{ Mean $\pm S D$} \\
\hline & Controls $(n=69)$ & $\begin{array}{l}\text { Diabetes mellitus without } \\
\text { nephropathy }(n=53)\end{array}$ & $\begin{array}{l}\text { Diabetes mellitus with } \\
\text { nephropathy }(n=51)\end{array}$ \\
\hline Type of diabetes mellitus & - & 2 & 2 \\
\hline Age (years) & $52.3 \pm 11.3$ & $51.2 \pm 13.1$ & $53.5 \pm 9.7^{*}$ \\
\hline Sex (male/female) & $36 / 33$ & $29 / 24$ & $27 / 24^{*}$ \\
\hline BMI $\left(\mathrm{kg} / \mathrm{m}^{2}\right)$ & $23.0 \pm 0.8$ & $25.1 \pm 1.1$ & $26.3 \pm 1.4^{*}$ \\
\hline Smokers \% & - & 42 & $42 *$ \\
\hline Duration of diabetes mellitus discovered & - & $6.7 \pm 1.8$ & $8.1 \pm 2.1$ \\
\hline
\end{tabular}

The values are mean $\pm \mathrm{SD},{ }^{*} \mathrm{p}<0.001$, highly significantly compared to controls. BMI: Body mass index, HTN: Hypertension, SD: Standard deviation

Table 2: Biochemical parameters of study subjects

\begin{tabular}{|c|c|c|c|}
\hline \multirow[t]{2}{*}{ Parameters } & \multicolumn{3}{|l|}{ Mean \pm SD } \\
\hline & Controls $(n=69)$ & $\begin{array}{l}\text { Diabetes mellitus without } \\
\text { nephropathy }(n=53)\end{array}$ & $\begin{array}{l}\text { Diabetes mellitus with } \\
\text { nephropathy }(n=51)\end{array}$ \\
\hline Fasting blood glucose (mg/dl) & $93.0 \pm 9.1$ & $173.0 \pm 6.3^{*}$ & $202.2 \pm 9.8^{* *}$ \\
\hline $\mathrm{HbA} 1 \mathrm{c} \%$ & $4.4 \pm 0.6$ & $7.5 \pm 1.2 *$ & $8.2 \pm 1.7^{* *}$ \\
\hline Urea (mg/dl) & $18.2 \pm 2.1$ & $28.9 \pm 3.2^{*}$ & $62.0 \pm 4.2^{* *}$ \\
\hline Creatinine (mg/dl) & $0.8 \pm 0.3$ & $1.0 \pm 0.5^{*}$ & $2.6 \pm 1.2^{* *}$ \\
\hline Microalbuminuria (mg/24 h) & $8.71 \pm 2.1$ & $13.23 \pm 4.1^{*}$ & $98.51 \pm 40.2^{* *}$ \\
\hline
\end{tabular}

The values are mean \pm SD; ANOVA test was done for multiple comparisons and Student's t-test for significance between the groups. * $\mathrm{p}<0.001$, control versus diabetes mellitus and ${ }^{* *} \mathrm{p}<0.001$, diabetes mellitus versus diabetes with nephropathy. HbA1c: Glycosylated hemoglobin, SD: Standard deviation

Table 3: Activities of antioxidant enzymes and MDA concentration in study subjects

\begin{tabular}{lll}
\hline Parameters & Mean \pm SD & \\
\cline { 2 - 3 } & Controls(n=69) & $\begin{array}{l}\text { Diabetes mellitus without } \\
\text { nephropathy (n=53) }\end{array}$ \\
\hline GPx (U/g Hb) & $28.2 \pm 8.1$ & $22.4 \pm 5.0^{*}$ \\
SOD (U/g Hb) & $6.9 \pm 0.6$ & $5.1 \pm 0.5^{*}$ \\
Catalase (U/g Hb) & $8.2 \pm 1.8$ & $6.5 \pm 1.0^{*}$ \\
MDA (nmol/ml) & $3.8 \pm 0.9$ & $6.9 \pm 1.8^{*}$ \\
\hline
\end{tabular}

The values are mean \pm SD; ANOVA test was done for multiple comparisons and Student's t-test for significance between the groups * $<<0.001$, control versus diabetes mellitus and ${ }^{* *} \mathrm{p}<0.001$, diabetes mellitus versus diabetes with nephropathy. GPx: Glutathione peroxidase, SOD: Superoxide dismutase, MDA: Malondialdehyde, SD: Standard deviation 
Table 4: Correlation analysis of HbA1c levels between diabetes mellitus with and without nephropathy patients

\begin{tabular}{lll}
\hline Parameters & $\begin{array}{l}\text { Diabetes mellitus } \\
\text { without nephropathy " } r \text { " }\end{array}$ & $\begin{array}{l}\text { Diabetes mellitus } \\
\text { with nephropathy “ } r \text { " }\end{array}$ \\
\hline HbA1c/GPx & $-0.73^{*}$ & $-0.79^{*}$ \\
HbA1c/SOD & $-0.52^{*}$ & $-0.61^{*}$ \\
HbA1c/CTL & $-0.68^{*}$ & $-0.74^{*}$ \\
HbA1c/MDA & $0.78^{*}$ & $0.85^{*}$ \\
\hline
\end{tabular}

*Pearson correlation coefficient is significant at $\mathrm{p}<0.05$, GPx: Glutathione peroxidase, SOD: Superoxide dismutase, MDA: Malondialdehyde, CTL: Catalase, HbA1c: Glycosylated hemoglobin

Once microalbuminuria persist, both changes in glomerular structure, such as mesangial expansion, basement membrane thickening, and permeability happened, which is referred as "incipient nephropathy." Diabetic subjects with persistent microalbuminuria are at increased risk for "over DN." At this stage, prominent proteinuria, hypertension, and renal insufficiency progressed. The pathological findings in this stage are glomerular basement membrane, thickening, mesangial expansion, and resulting in diffuse and/or nodular glomerulosclerosis, afferent and efferent arteriolar hyalinosis, and tubulointerstitial fibrosis. After several years of persistent proteinuria, the progression of ESRD will occur [22]. Recent evidence suggests that glomerular podocytes have a key role in early proteinuria, and reduced podocyte number was reported in patients with DN. Experimental studies suggest that amelioration of excessive oxidative stress by antioxidant therapies may potentially prevent glomerular and podocyte damage in DN [23].

Like other previous studies [24], we demonstrated that hypertension and obesity were found to be significantly higher in T2DM patients and further increased in T2DM with nephropathy patients. Tobacco smoking is one of the most important modifiable risk factors for the development of so many diseases. The smoking habit, concurrent with multiple metabolic toxicities of DM and metabolic syndrome, likely accelerates the multifaceted pathogenetic mechanisms of diabetic glomerulosclerosis and chronic kidney disease [25]. Cigarette smoke contains so many free radicals in both the gas and tar phases, and it decreases the total antioxidant capacity of several critical enzymes, which are involved in the free radical detoxification, including GPx, SOD, metallothionein, and thioredoxin reductase [26]. In the present study, we observed more smokers in T2DM and T2DM with nephropathy compared to controls. Interestingly, a new study from China has shown that the expansion of visceral adiposity is a risk factor for an elevated risk of $24 \mathrm{~h}$ urinary albumin excretion, and with the expansion of visceral adiposity, the prevalence of heavy albuminuria increases in DN patients [27]. Our study indicated that there is an increase of urine microalbuminuria in T2DM patients, and further, elevation was observed in T2DM with nephropathy patients. Vandana Varma et al. [28] and Hanai et al. [29], in their experiments and patient's studies, have shown similar results of urine microalbuminuria. Then, the findings indicate that protein, lipid, and DNA damage already may exist in the early stages of DN in patients with T2DM and that the levels of oxidative stress increase as microalbuminuria becomes more pronounced. These studies confirmed that microalbuminuria was currently an excellent nontraumatic predictor of the early stage of DN.

MDA is a natural product of LPO and reflects the oxidant status of the biological system. LPO is a free radical linked chain reaction and which is self-perpetuating. Several authors in their experimental and patients studies have revealed that increased levels of serum MDA in DN patients [5,30]. In our study, we also observed the increased levels of MDA in T2DM and further increased in T2DM patients with nephropathy as compared to controls, possibly because there is an increased production of ROS in the circulation. This fact may indicate increased production of free radicals or diminished efficiency of antioxidant defense mechanisms in DN patients [31]. The estimation of LPO in the patients with DN is very useful to judge the progress of the patient.
Human body combat against the free radicals by its natural defense with antioxidant enzymes and exogenous antioxidants. There are many enzymatic antioxidants in vivo such as GPx, SOD, and CTL that play a very important role in the protection against LPO. Phospholipid hydroperoxide GPx, an intrinsic renal cytoprotective antioxidant enzyme mainly expressed in glomerular podocytes, parietal epithelial cells, as well as tubular epithelial cells play a prominent role in preventing LPO [32]. The enzyme reduced glutathione reduces the oxidized form of the enzyme GPx, which in turn reduces $\mathrm{H}_{2} \mathrm{O}_{2}$, a dangerously reactive species within the cell. The increased production of LPO and $\mathrm{H}_{2} \mathrm{O}_{2}$ resulting a decreased consumption of GSH, this impairs the depletion GPx activity in DN patients. Increased activity of aldolase reductase pathway in patients with DN consumes NADPH, resulting in impaired the activity of GR. Thus, decreased GPx antioxidant enzyme was seen in patients with DN [33]. A several authors reported a decreased activity of GPx in DN patients [2,34]. Similarly, we also observed a decreased activity of GPx in DN patients compared to controls. The resultant oxidative damage can then contribute to the pathogenesis of DN.

Another one of the important antioxidant enzymes in the human body is SOD. Some authors have reported a decreased level of SOD in the blood [31,35]. We have also found a decrease in SOD levels in T2DM patients, and further decreased activity was observed in T2DM patients with nephropathy. Kimura et al. [36] and Moussa et al. [37] in their study displayed increased SOD levels in patients with DM. The decrease in serum SOD activity in T2DM is linked to be progressive glycation of enzymatic proteins. About $50 \%$ of SOD in erythrocytes of diabetic patients is glycated, resulting in trace activity. Whereas further lower activity of SOD levels in DN patients could be due to excessive production of superoxide radicals, consumption in the autoxidation procedure and increased excretion from inflammatory kidney in nephropathy [38]. CTL is apparently the most efficient enzyme found in our cells [39]. Selvam et al. [40] reported an increase in CTL activity in T2DM with nephropathy patients. On the other hand, Inah Hwang et al. [41] revealed that a diabetic person had decreased activity in CTL. Similarly, our study showed that CTL levels were found significantly reduced in T2DM patients with nephropathy. Increased ROSs in CTL deficiency impair peroxisomal/mitochondrial fatty acid oxidation and subsequently increase lipid accumulation, which might induce increased renal injury [42]. Animal and clinical studies have confirmed that antioxidant treatment plays an effective role in T2DM and DN [43].

There are many evidence suggest that ROS plays an important role in the pathogenesis of DN. To prevent the development and progression of DN, it would be effective in combing the strategies to prevent overproduction of ROS and to increase the removal of performed ROS. Some natural antioxidants were proved to process the ability to decelerate DN through reducing oxidative status. The flower of Hibiscus sabdariffa Linnaeus calyx is commonly used in hot and cold beverages and as a supplement due to its perceived potential of health benefits. The flower extract has been reported to decrease blood pressure and has antitumor characteristics as well as immune-modulating and antileukemic effects, and also, extract contains polyphenolic acids, flavonoids, and protocatechuic acids [44]. Wang et al. [45] demonstrated that aqueous extract of $H$. sabdariffa Linnaeus is capable of increasing CTL and GSH enzyme activities significantly in diabetic kidney disease. Another, Luteolin is a plantderived flavonoid that has protecting effect against the development of DN by increasing the SOD activity, decreasing MDA content, and expression of heme oxygenase- 1 protein [46]. Some authors revealed that antioxidants such as a lipoic acid, propolis, astaxanthin, flax, and pumkin seed mixture have been proved as an important treatment regimens for diabetic renal disease to reduce oxidative stress [18]. However, further clinical studies are needed to illuminate their therapeutic potential in treating diabetic patients with nephropathy.

Some clinical evidence studies showed that the improvement of albuminiuria, HbA1c, MDA in T2DM patients with combined using of antioxidants therapy or antioxidant with anti-inflammation agent [47]. Recently, one report hypothesizez that natural substances have 
antioxidant and anti-inflammatory activities and have an impact on NFB and KEAP1/Nrf2/ARE pathways. The preclinical studies explored the effectiveness of whole herbs, plants, or seeds and their active ingredients that might change our strategies for the management of DN [48]. In future studies, the new antioxidants will be preferred which show multiple effects against DN pathogenesis pathways. There were some limitations in the present study, our sample size was small, and since it was a hospital-based study, so we cannot represent over the entire population. This is needed to be proven in clinical trial studies.

\section{CONCLUSION}

This will be the first report to measure oxidants and particularly key antioxidant enzymes in T2DM patients with nephropathy among the Saudi population in Al-Quwayiyah region of Saudi Arabia; there are taking more junk food, animal saturated fat, and high cholesterol food all these affect for so many diseases. However, they are not very well aware of their DM, kidney disease, and cardiovascular diseases. In an earlier stage of DM patients, they want to take some important precautions, otherwise this may affect as a key initiator of kidney damage associated with DN. Oxidative stress coupled with chronic hyperglycemia may have an important role in the pathogenesis of glomerular and tubular functional and structural abnormalities. The study illustrated that continuous use of antioxidants with long half-life time; it increases of antioxidant concentration in specific cellular organelle such as mitochondrion, show anti-inflammatory, blood lowering pressure effect, and increase body antioxidant enzyme activity. Hence, monitoring of these antioxidant enzymes and microalbuminuria parameters in the early stage of patients with T2DM could be vital importance in possible preventing further development of complications. We suggest that potential and new multiproperty antioxidant therapy seems to be promising in preventing the induction and progression of DN before reaching to ESRD.

\section{REFERENCES}

1. Matough FA, Budin SB, Hamid ZA, Alwahaibi N, Mohamed J. The role of oxidative stress and antioxidants in diabetic complications. Sultan Qaboos Univ Med J 2012;12:5-18

2. Kumawat M, Sharma TK, Singh I, Singh N, Ghalaut VS, Vardey SK, et al. Antioxidant enzymes and lipid peroxidation in Type 2 diabetes mellitus patients with and without nephropathy. N Am J Med Sci 2013;5:213-9.

3. Gnudi L. Cellular and molecular mechanisms of diabetic glomerulopathy. Nephrol Dial Transplant 2012;27:2642-9.

4. Shao N, Kuang HY, Wang N, Gao XY, Hao M, Zou W, et al. Relationship between oxidant/Antioxidant markers and severity of microalbuminuria in the early stage of nephropathy in Type 2 diabetic patients. J Diabetes Res 2013;2013:232404.

5. Wolf G, Thaiss F. Hyperglycaemia-Pathophysiological aspects at the cellular level. Nephrol Dial Transplant 1995;10:1109-12.

6. Stehouwer CD. Endothelial dysfunction in diabetic nephropathy: State of the art and potential significance for non-diabetic renal disease. Nephrol Dial Transplant 2004;19:778-81

7. Mahbood MF, Rahman PG. Serum lipid peroxidation and antioxidant enzyme levels in male and female diabetic subjects. Singapore Med J 2005;46:322-4.

8. Tavafi M. Diabetic nephropathy and antioxidants. J Nephropathol 2013;2:20-7

9. World health Organization. Definition and diagnosis of diabetes mellitus and intermediate hyperglycemia: Report of a WHO/IDF consultation. World health Organization; 2006. p. 1-46.

10. Mogensen CE, Christensen CK, Vittinghus E. The stages in diabetic renal disease. With emphasis on the stage of incipient diabetic nephropathy. Diabetes 1983;32 Suppl 2:64-78.

11. Trinder P. Blood sugar estimation by GOD- POD method. Ann Clin Biochem 1969;6:24-7.

12. Karl J, Burns G, Engel WD, Finke A, Krantzer M, Rolliner W, et al. Development and standardization of a new immunoturbidimetric HbA1c assay. Clin Lab 1993;39:991-6.

13. Gey KF, Brubacher GB, Stähelin HB. Plasma levels of antioxidant vitamins in relation to ischemic heart disease and cancer. Am J Clin Nutr 1987;45:1368-77

14. Paglia DE, Valentine WN. Studies on the quantitative and qualitative characterization of erythrocyte glutathione peroxidase. J Lab Clin Med 1967:70:158-69.

15. Arthur JR, Boyne R. Superoxide dismutase and glutathione peroxidase activities in neutrophils from selenium deficient and copper deficient cattle. Life Sci 1985;36:1569-75.

16. Beers RF Jr, Sizer IW. A spectrophotometric method for measuring the breakdown of hydrogen peroxide by catalase. J Biol Chem 1952;195:133-40.

17. Yagi K. Lipid peroxides and human disease. Chem Phy lipids 1987;45:337-51

18. Singh N, Sharma P, Garg V, Mondal S, Singh A. Antioxidant therapy in diabetic nephropathy. J Pharm Res 2011;4:4249-51.

19. Giacco F, Brownlee M. Oxidative stress and diabetic complications. Circ Res 2010;107:1058-70.

20. Krishan P, Chakkarwar VA. Diabetic nephropathy: Aggressive involvement of oxidative stress. J Pharm Edu Res 2011;2:35-41.

21. Park IS, Kiyomoto H, Abboud SL, Abboud HE. Expression of transforming growth factor-beta and Type IV collagen in early streptozotocin-induced diabetes. Diabetes 1997;46:473-80.

22. Lee WC, Wang CJ, Lee HJ. Antioxidants in decelerating diabetic nephropathy. Intech. DOI: 10.5772/45927; 387-399.

23. Spurney RF, Coffman TM. Stressed-out podocytes in diabetes? J Am Soc Nephrol 2008;19:2035-7.

24. Zimmerman FH, Cameron A, Fisher LD, Ng G. Myocardial infarction in young adults: Angiographic characterization, risk factors and prognosis (Coronary artery surgery study registry). J Am Coll Cardiol 1995;26:654-61.

25. Baggio B, Budakovic A, Dalla Vestra M, Saller A, Bruseghin M, Fioretto P, et al. Effects of cigarette smoking on glomerular structure and function in Type 2 diabetic patients. J Am Soc Nephrol 2002;13:2730-6.

26. Yoshida T, Tuder RM. Pathobiology of cigarette smoker induced chronic obstructive pulmonary disease. Physiol Rev 2007;87:1047-82.

27. Zhai S, Wang ZJ. Association of visceral adiposity with urinary albumin excretion in Type 2 diabetics. Zhonghua Yi Xue Za Zhi 2011;91:2108-11.

28. Varma V, Varma M, Sarkar PD, Varma A, Vyas S, Kulkarani R. Correlation of vitamin $\mathrm{C}$ with $\mathrm{HbA} 1 \mathrm{c}$ and oxidative stress in diabetes mellitus with or without nephropathy. Natl J Med Res 2014;4:151-5.

29. Hanai K, Babazono T, Nyumura I, Toya K, Ohta M, Bouchi R, et al. Involvement of visceral fat in the pathogenesis of albuminuria in patients with Type 2 diabetes with early stage of nephropathy. Clin Exp Nephrol 2010;14:132-6.

30. Gupta MM, Chari S. Lipid peroxidation and antioxidant status in patients with diabetic retinopathy. Indian J Physiol Pharmacol 2005:49:187-92.

31. Kedziora-Kornatowska KZ, Luciak M, Blaszczyk J, Pawlak W. Lipid peroxidation and activities of antioxidant enzymes in erythrocytes of patients with non-insulin dependent diabetes with or without diabetic nephropathy. Nephrol Dial Transplant 1998;13:2829-32.

32. Conz PA, Bevilacqua PA, LaGreca G, Danieli D, Rodighiero MP, Cavarretta L, et al. Phospholipid hydroperoxidase glutathione peroxidase in the normal human kidney: A possible role in protecting cell membranes. Exp Nephrol 1993;1:376-8.

33. Bhatia S, Shukla R, Venkata Madhu S, Kaur Gambhir J, Madhava Prabhu K. Antioxidant status, lipid peroxidation and nitric oxide end products in patients of Type 2 diabetes mellitus with nephropathy. Clin Biochem 2003;36:557-62.

34. Waggiallah H, Alzohairy M. The effect of oxidative stress on human red cells glutathione peroxidase, glutathione reductase level, and prevalence of anemia among diabetics. N Am J Med Sci 2011;3:344-7.

35. Sundaram RK, Bhaskar A, Vijayalingam S, Viswanathan M, Mohan R, Shanmugasundaram KR, et al. Antioxidant status and lipid peroxidation in Type II diabetes mellitus with and without complications. Clin Sci (Lond) 1996;90:255-60

36. Kimura F, Hasegawa G, Obayashi H, Adachi T, Hara H, Ohta M, et al. Serum extracellular superoxide dismutase in patients with Type 2 diabetes: Relationship to the development of micro- and macrovascular complications. Diabetes Care 2003;26:1246-50.

37. Moussa SA. Oxidative stress in diabetes mellitus. Rom J Biophys 2008;18:225-36.

38. Arai K, Lizuka S, Tada Y, Oilkawa K, Taniguelui N. Increase in the glycosylated form of erythrocyte $\mathrm{Cu} \mathrm{Zn}$ SOD diabetes and association of non-enzymatic glycosylation with enzyme activity. Biochem Biophys Acta 1987;924:292-6.

39. Gerli GC, Beretta L, Bianchi M, Pellegatta A, Agostoni A. Erythrocyte superoxide dismutase, catalase and glutathione peroxidase activities in beta-thalassaemia (major and minor). Scand J Haematol 1980;25:87-92.

40. Selvam R, Anuradha CV. Lipid peroxidation and antiperoxidative 
enzyme changes in erythrocytes in diabetes mellitus. Indian J Biochem Biophys 1988;25:268-72.

41. Hwang I, Lee J, Huh JY, Park J, Lee HB, Ho YS, et al. Catalase deficiency accelerates diabetic renal injury through peroxisomal dysfunction. Diabetes 2012;61:728-38.

42. Tilton WM, Seaman C, Carriero D, Piomelli S. Regulation of glycolysis in the erythrocyte: Role of the lactate/pyruvate and NAD/NADH ratios. J Lab Clin Med 1991;118:146-52.

43. Ogawa S, Nako K, Okamura M, Senda M, Mori T, Ito S, et al. Aliskiren reduces albuminuria and oxidative stress, and elevates glomerular filtration rates in Japanese patients with advanced diabetic nephropathy. Hypertens Res 2011;34:400-1

44. Tseng TH, Kao TW, Chu CY, Chou FP, Lin WL, Wang CJ, et al. Induction of apoptosis by hibiscus protocatechuic acid in human leukemia cells via reduction of retinoblastoma $(\mathrm{RB})$ phosphorylation and bcl-2 expression. Biochem Pharmacol 2000;60:307-15.
45. Wang SC, Lee SF, Wang CJ, Lee CH, Lee WC, Lee HJ. Aqueous Extract from Hibiscus sabdariffa Linnaeus ameliorate diabetic nephropathy via regulating oxidative status and $\mathrm{Akt} / \mathrm{Bad} / 14-3-3$ \{gamma in an experimental animal model. Evid-Based Complement Alternat Med 2011;938126. DOI: 10.1093/ecam/nep181. Epub 2011 Feb 20.

46. Wang GG, Lu XH, Li W, Zhao X, Zhang C. Protective effects of luteolin on diabetic nephropathy in STZ-induced diabetic rats. Evid Based Complement Alternat Med 2011;2011:323171.

47. Fallahzadeh MK, Dormanesh B, Sagheb MM, Roozbeh J, Vesssal G, Pakfetrat M. Effect of addition of silymarin to rennin-angiotension system inhibitors on proteinuria in Type 2 diabetic patients with overt nephropathy: A randomized, double-blind, placebo-controlled trail. Am J Kidney Dis 2012;60:896-903

48. Al-Waili N, Al-Waili H, Al-Waili T, Salom K. Natural antioxidants in the treatment and prevention of diabetic nephropathy; a potential approach that warrants clinical trials. Redox Rep 2017;22:99-118. 